Stud. Univ. Babeş-Bolyai Math. 64(2019), No. 3, 497-510

DOI: 10.24193/subbmath.2019.4.05

\title{
On the periodicity of meromorphic functions when sharing two sets IM
}

\author{
Molla Basir Ahamed
}

\begin{abstract}
In this paper, we have considered two sets sharing problems, and investigated on some sufficient conditions for the periodicity of meromorphic functions and obtained two results improving the result of Bhoosnurmath-Kabbur [6], QiDou-Yang [17] and Zhang [20]. The results are:

Let $\mathcal{S}_{1}=\left\{z: \int_{0}^{z-a}(t-a)^{n}(t-b)^{4} d t+1=0\right\}$ and $\mathcal{S}_{2}=\{a, b\}$, where $n \geq 4(n \geq 2)$ be an integer. Let $f(z)$ be a non-constant meromorphic (entire) function satisfying $\bar{E}_{f(z)}\left(\mathcal{S}_{j}\right)=\bar{E}_{f(z+c)}\left(\mathcal{S}_{j}\right),(j=1,2)$ then $f(z) \equiv f(z+c)$. Some examples have been exhibited to show that, the meromorphic functions, we have considered may be of infinite order, and also to show that the sets considered in the main results, can't be replace by some arbitrary sets. At the last section, we have posed a question for the future research in this direction.
\end{abstract}

Mathematics Subject Classification (2010): 30D35.

Keywords: Meromorphic function, shared sets, finite and infinite order, shift operator, periodicity.

\section{Introduction}

We assume that the reader is familiar with the elementary Nevanlinna theory, see, e.g., $[11,13,14,18]$. Meromorphic functions are always non-constant, unless otherwise specified. For such a function $f$ and $a \in \overline{\mathbb{C}}=: \mathbb{C} \cup\{\infty\}$, each $z$ with $f(z)=a$ will be called $a$-point of $f$. We will use here some standard definitions and basic notations from this theory. In particular by $N(r, a ; f)(\bar{N}(r, a ; f))$ we denote the counting function (reduced counting function) of $a$-points of meromorphic functions $f, T(r, f)$ is the Nevanlinna characteristic function of $f$ and $S(r, f)$ is used to denote each functions which is of smaller order than $T(r, f)$ when $r \rightarrow \infty$.

We also denote $\mathbb{C}^{*}:=\mathbb{C} \backslash\{0\}$. As for the standard notation in the uniqueness theory of meromorphic functions, suppose that $f$ and $g$ are meromorphic. Denoting 
$E_{f}(a)\left(\bar{E}_{f}(a)\right)$, the set of all $a$-points of $f$ counting multiplicities (ignoring multiplicities). We say that two meromorphic functions $f, g$ share the value $a C M(I M)$ if $E_{f}(a)=E_{g}(a)\left(\bar{E}_{f}(a)=\bar{E}_{g}(a)\right)$.

The classical results in the uniqueness theory of meromorphic functions are the five-point, resp. four-point, theorems due to Nevanlinna [16]: If two meromorphic functions $f, g$ share five distinct values in the extended complex plane $I M$, then $f \equiv g$. The beauty of this result lies in the fact that there is no counterpart of this result in the real function theory. Similarly, if two meromorphic functions $f, g$ share four distinct values in the extended complex plane $C M$, then $f \equiv T \circ g$, where $T$ is a Möbius transformation.

Clearly these results initiated the study of uniqueness of two meromorphic functions $f$ and $g$. The study becomes more interesting if the function $g$ is related with $f$.

Definition 1.1. For a non-constant meromorphic function $f$ and any set $\mathcal{S} \subset \overline{\mathbb{C}}$, we define

$$
\begin{aligned}
E_{f}(\mathcal{S})= & \bigcup_{a \in \mathcal{S}}\{(z, p) \in \mathbb{C} \times \mathbb{N}: f(z)=a, \text { with multiplicity } p\}, \\
& \bar{E}_{f}(\mathcal{S})=\bigcup_{a \in \mathcal{S}}\{(z, 1) \in \mathbb{C} \times\{1\}: f(z)=a\} .
\end{aligned}
$$

If $E_{f}(\mathcal{S})=E_{g}(\mathcal{S})\left(\bar{E}_{f}(\mathcal{S})=\bar{E}_{g}(\mathcal{S})\right)$ then we simply say $f$ and $g$ share $\mathcal{S}$ Counting Multiplicities(CM) (Ignoring Multiplicities(IM)).

Evidently, if $\mathcal{S}$ contains one element only, then it coincides with the usual definition of $C M(I M)$ sharing of values.

Definition 1.2. For a non-constant meromorphic function $g$ and $a \in \mathbb{C}$, we define $\bar{N}_{(2}\left(r, \frac{1}{g-a}\right)$ the reduced counting function of those $a$-points of $g$ of multiplicities $\geq 2$.

In 1976, Gross [12] precipitated the research instigating the set sharing problem with a more general set up made tracks various direction of research for the uniqueness theory.

In connection with the question posed by Gross in[12], a sprinkling number of results have been obtained by many mathematicians $[2,3,5,9,19,21]$ concerning the uniqueness of meromorphic functions sharing two sets. But in most of the preceding results, in the direction, one set has always been kept fixed as the set of poles of a meromorphic function.

Recently set sharing corresponding to a function and its shift or difference operator have been given priority by the researchers than that of the introductory one.

In what follows, $c$ always means a non-zero constant. For a non-constant meromorphic function, we define its shift and difference operator respectively by $f(z+c)$ and $\Delta_{c} f=f(z+c)-f(z)$.

Now-a-days among the researchers $[1,4,6,7,8,17,20]$, an increasing amount of interest has been found to find the possible relationship between a meromorphic function $f(z)$ and its shift $f(z+c)$ or its difference $\Delta_{c} f$. 
At the earlier stage, several authors were devoted to find uniqueness problems between two meromorphic functions $f$ and $g$ sharing two sets. But in this particular direction, the first inspection for uniqueness of a meromorphic function and its shift was due to Zhang [20].

In 2010, Zhang [20] obtained the following results.

Theorem A. [20] Let $m \geq 2, n \geq 2 m+4$ with $n$ and $n-m$ having no common factors. Let $a$ and $b$ be two non-zero constant such that the equation $w^{n}+a w^{n-m}+b=0$ has no multiple roots. Let $\mathcal{S}_{1}=\left\{w: w^{n}+a w^{n-m}+b=0\right\}$ and $\mathcal{S}_{2}=\{\infty\}$. Suppose that $f(z)$ is a non-constant meromorphic function of finite order. Then $E_{f(z)}\left(\mathcal{S}_{j}\right)=E_{f(z+c)}\left(\mathcal{S}_{j}\right)$ $(j=1,2)$ imply that $f(z) \equiv f(z+c)$.

Remark 1.1. For meromorphic function, note that $\#\left(\mathcal{S}_{1}\right)=9$ when the nature of sharing is $C M$.

Theorem B. [20] Let $n \geq 5$ be an integer and let $a, b$ be two non-zero constants such that the equation $w^{n}+a w^{n-1}+b=0$ has no multiple roots.

Denote $\mathcal{S}_{1}=\left\{w: w^{n}+a w^{n-1}+b=0\right\}$. Suppose that $f$ is a non-constant entire function of finite order. Then $E_{f(z)}\left(\mathcal{S}_{1}\right)=E_{f(z+c)}\left(\mathcal{S}_{1}\right)$ implies $f(z) \equiv f(z+c)$.

Remark 1.2. For entire function, note that $\#\left(\mathcal{S}_{1}\right)=5$, when the nature of sharing is $C M$.

Thus we see that Zhang obtained the results for meromorphic function with the cardinality of main range set as 9 and for entire function as 5 .

Later, Qi-Dou-Yang [17] studied the case for $m=1$ in Theorem $A$ and with the aid of some extra supposition and got $\#\left(\mathcal{S}_{1}\right)=6$ when the nture of sharing is $C M$.

Afterworlds, Bhoosnurmath-Kabbur [6] improved Theorem $A$ by reducing the lower bound of the cardinality of range set in a little different way and obtained the following result.

Theorem C. [6] Let $n \geq 8$ be an integer and $c(\neq 0,1)$ is a constant such that the equation

$$
P(w)=\frac{(n-1)(n-2)}{2} w^{n}-n(n-2) w^{n-1}+\frac{n(n-1)}{2} z^{n-2}-c .
$$

Let us suppose that $S_{1}=\{w: P(w)=0\}$ and $S_{2}=\{\infty\}$. Suppose that $f(z)$ is a non-constant meromorphic function of finite order. Then $E_{f(z)}\left(\mathcal{S}_{j}\right)=E_{f(z+c)}\left(\mathcal{S}_{j}\right)$ $(j=1,2)$ imply that $f(z) \equiv f(z+c)$.

Remark 1.3. For meromorphic function, we see that $\#\left(\mathcal{S}_{1}\right)=8$ when the nature of sharing is $C M$.

The worth noticing fact is that, the lower bound of the cardinality of the main range set for the meromorphic function has always been fixed to 8 without the help of any extra supposition.

So for the improvement of all the above mentioned results it is quite natural to investigate in this direction. Theorems $A, B, C$ really motivates oneself for further study in this direction by solving the following question. 
Question 1.1. Is it possible to diminish further the lower bound of the cardinalities of the main range sets in Theorems $A, B$ and $C$ ?

We also note that no attempts have so far been made by any researchers, till now to the best of our knowledge, to relax the nature of sharing the sets in connection with the periodicity of a meromorphic function when sharing sets. So the following question is inevitable.

Question 1.2. Can we relax the nature of sharing the sets from $C M$ to $I M$ in Theorems $A, B$ and $C$ ?

It would be interesting to know what happens if we replace the set of poles $\{\infty\}$ by new set in Theorems $A, B, C$.

In all the above mentioned results, the respective authors have considered meromorphic function with finite ordered and got their results. So a natural investigation is that: Are Theorems $A, B, C$ not valid for infinite ordered meromorphic function ?

The following examples show that Theorems $A, B, C$ are true for infinite ordered meromorphic functions also.

Example 1.1. Let

$$
f(z)=\frac{\exp \left(\exp \left(\frac{2 \pi i z}{c}\right)\right)}{\exp \left(\frac{2 \pi i z}{c}\right)-1} .
$$

Clearly $f(z)$ and $f(z+c)$ share the corresponding sets $\mathcal{S}_{1}$ and $\mathcal{S}_{2}$ in Theorems $A, B$, $C$, and $f(z) \equiv f(z+c)$.

Example 1.2. Let

$$
f(z)=\frac{\exp \left(\sin \left(\frac{2 \pi z}{c}\right)\right)}{\tan \left(\frac{\pi z}{c}\right)-1} .
$$

Evidently, $f(z)$ and $f(z+c)$ share the corresponding sets $\mathcal{S}_{1}$ and $\mathcal{S}_{2}$ in Theorems $A$, $B, C$, and $f(z) \equiv f(z+c)$.

One can construct such examples plenty in numbers. Therefore, one natural question arises as follows:

Question 1.3. Can we get a corresponding results like Theorems $A, B, C$ by omitting the term finite ordered?

\section{Main results}

Answering all the questions affirmatively is the main motivation of writing this paper. Throughout the paper, for an integer $n \geq 4$, we will denote by

$$
\mathcal{P}(z)=\int_{0}^{z-a}(t-a)^{n}(t-b)^{4} d t+1, \text { where } a, b \in \mathbb{C} \text { with } a \neq b .
$$

Following are the two main result of this paper. 
Theorem 2.1. Let $\mathcal{S}_{1}=\{z: \mathcal{P}(z)=0\}$ and $\mathcal{S}_{2}=\{a, b\}$, where $a \in \mathbb{C}^{*}, n \geq 4$ be an integer. If $f(z)$ be a non-constant meromorphic function satisfying

$$
\bar{E}_{f(z)}\left(\mathcal{S}_{j}\right)=\bar{E}_{f(z+c)}\left(\mathcal{S}_{j}\right),(j=1,2)
$$

then $f(z) \equiv f(z+c)$.

Remark 2.1. For non-entire meromorphic function, one may observe that $\#\left(\mathcal{S}_{1}\right)=9$ when the nature of sharing is $I M$.

Theorem 2.2. Let $\mathcal{S}_{1}=\{z: \mathcal{P}(z)=0\}$ and $\mathcal{S}_{2}=\{a, b\}$, where $a \in \mathbb{C}^{*}, n \geq 2$ be an integer. If $f(z)$ be a non-constant entire function satisfying $\bar{E}_{f(z)}\left(\mathcal{S}_{j}\right)=\bar{E}_{f(z+c)}\left(\mathcal{S}_{j}\right)$, $(j=1,2)$, then $f(z) \equiv f(z+c)$.

Remark 2.2. For entire function, we see that $\#\left(\mathcal{S}_{1}\right)=7$ when the nature of sharing is $I M$.

The following examples satisfy Theorems 2.1 and 2.2 for "entire" as well as "meromorphic" functions.

Example 2.1. Let us suppose that

$$
f(z)=\frac{\tan \left(\frac{\pi z}{c}\right)+\alpha}{\tan \left(\frac{\pi z}{c}\right)-\beta}+\frac{\cos \left(\frac{2 \pi z}{c}\right)+\gamma}{\sin \left(\frac{2 \pi z}{c}\right)-\delta}
$$

where $\alpha, \beta, \gamma, \delta, c \in \mathbb{C}^{*}$. It is clear that $\bar{E}_{f(z)}\left(\mathcal{S}_{j}\right)=\bar{E}_{f(z+c)}\left(\mathcal{S}_{j}\right),(j=1,2)$ in Theorem 2.1 and note that $f(z) \equiv f(z+c)$.

Example 2.2. Let

$$
f(z)=\frac{\alpha+\beta \sin ^{2}\left(\frac{\pi z}{c}\right)}{\gamma-\delta \cos ^{2}\left(\frac{\pi z}{c}\right)}
$$

where $p$ be an even positive integer, $\alpha, \beta, \gamma, \delta, c \in \mathbb{C}^{*}$.

It is clear that $\bar{E}_{f(z)}\left(\mathcal{S}_{j}\right)=\bar{E}_{f(z+c)}\left(\mathcal{S}_{j}\right),(j=1,2)$ in Theorem 2.1 and note that $f(z) \equiv f(z+c)$.

Example 2.3. Let

$$
f(z)=a e^{p z}+b \cos ^{2}\left(\frac{\pi z}{c}\right),
$$

where $p$ be an even positive integer, $a, b, c \in \mathbb{C}^{*}$ with $e^{c}=-1$. It is clear that $\bar{E}_{f(z)}\left(\mathcal{S}_{j}\right)=\bar{E}_{f(z+c)}\left(\mathcal{S}_{j}\right),(j=1,2)$ in Theorem 2.2 and note that $f(z) \equiv f(z+c)$.

The next examples shows that the set considered in Theorem 2.1 for "entire" and Theorem 2.2 for "meromorphic" functions respectively can not be replaced by arbitrary sets. 
Example 2.4. Let us suppose that $\mathcal{S}_{1}=\left\{\zeta: \zeta^{9}-1=0\right\}$ and $\mathcal{S}_{2}=\{0, \infty\}$. Let

$$
f(z)=\frac{a e^{z}}{b-d \sin ^{2}\left(\frac{\pi z}{c}\right)} .
$$

It is clear that $\bar{E}_{f(z)}\left(\mathcal{S}_{j}\right)=\bar{E}_{f(z+c)}\left(\mathcal{S}_{j}\right),(j=1,2)$ in Theorem 2.1 with $e^{c}=\zeta$ and $a, b, c, d \in \mathbb{C}^{*}$ and note that $f(z) \not \equiv f(z+c)$.

Example 2.5. Let us suppose that $\mathcal{S}_{1}=\left\{\zeta: \zeta^{7}-1=0\right\}$ and $\mathcal{S}_{2}=\{0,1\}$. Let

$$
f(z)=\exp \left(\cos \left(\frac{\pi z}{c}\right)\right) \quad \text { or } \exp \left(\sin \left(\frac{\pi z}{c}\right)\right) \text {. }
$$

Then $f(z+c)=\exp \left(-\cos \left(\frac{\pi z}{c}\right)\right)$ or $\exp \left(-\sin \left(\frac{\pi z}{c}\right)\right)$ respectively. It is clear that $\bar{E}_{f(z)}\left(\mathcal{S}_{j}\right)=\bar{E}_{f(z+c)}\left(\mathcal{S}_{j}\right),(j=1,2)$ in Theorem 2.2 and note that $f(z) \not \equiv f(z+c)$.

Example 2.6. Let

$$
\mathcal{S}_{1}=\left\{-1,1,-i, 0, i,-\frac{1}{\sqrt{2}}, \frac{1}{\sqrt{2}}\right\}
$$

and $\mathcal{S}_{2}=\{-2,2\}$. Let $f(z)=e^{z}$. It is clear that $\bar{E}_{f(z)}\left(\mathcal{S}_{j}\right)=\bar{E}_{f(z+c)}\left(\mathcal{S}_{j}\right),(j=1,2)$ in Theorem 2.2 with $e^{c}=-1, c \in \mathbb{C}^{*}$ and note that $f(z) \not \equiv f(z+c)$.

\section{Auxiliary definitions and some lemmas}

It was Fujimoto [10], who first discovered a special property of a polynomial, reasonably called as critical injection property though initially Fujimoto [10] called it as property $(\mathrm{H})$.

Definition 3.1. Let $\mathcal{P}(w)$ be a non-constant monic polynomial. We call $\mathcal{P}(w)$ a uniqueness polynomial if $\mathcal{P}(f) \equiv c \mathcal{P}(g)$ implies $f \equiv g$ for any non-constant meromorphic functions $f$ and $g$ and any non-zero constant $c$. We also call $\mathcal{P}(w)$ a uniqueness polynomial in a broad sense if $\mathcal{P}(f) \equiv \mathcal{P}(g)$ implies $f \equiv g$.

Next we recall here the property $(\mathrm{H})$ and critically injective polynomial. Let $\mathcal{P}(w)$ be a monic polynomial without multiple zero whose derivative has mutually distinct $k$-zeros $e_{1}, e_{2}, \ldots, e_{k}$ with the multiplicities $q_{1}, q_{2}, \ldots, q_{k}$ respectively.

Now, the property $\mathcal{P}\left(e_{l}\right) \neq \mathcal{P}\left(e_{m}\right)$ for $1 \leq l<m \leq k$ is a known as property $(\mathrm{H})$ and a polynomial $\mathcal{P}(w)$ satisfying this property is called critically injective polynomial.

Given meromorphic functions $f(z)$ and $f(z+c)$ we associate $\mathcal{F}, \mathcal{G}$ by

$$
\mathcal{F}=\mathcal{P}(f), \quad \mathcal{G}=\mathcal{P}(f(z+c)),
$$

to $\mathcal{F}, \mathcal{G}$ we associate $\mathcal{H}$ and $\Phi$ by the following formulas

$$
\mathcal{H}=\frac{\left(\frac{1}{\mathcal{F}}\right)^{\prime \prime}}{\left(\frac{1}{\mathcal{F}}\right)^{\prime}}-\frac{\left(\frac{1}{\mathcal{G}}\right)^{\prime \prime}}{\left(\frac{1}{\mathcal{G}}\right)^{\prime}}=\left(\frac{\mathcal{F}^{\prime \prime}}{\mathcal{F}^{\prime}}-\frac{2 \mathcal{F}^{\prime}}{\mathcal{F}}\right)-\left(\frac{\mathcal{G}^{\prime \prime}}{\mathcal{G}^{\prime}}-\frac{2 \mathcal{G}^{\prime}}{\mathcal{G}}\right)
$$




$$
\Phi=\frac{\mathcal{F}^{\prime}}{\mathcal{F}}-\frac{\mathcal{G}^{\prime}}{\mathcal{G}}
$$

Before proceeding to the actual proofs, we recall a few lemmas that take an important role in the reasoning.

Lemma 3.1. [15] Let $g$ be a non-constant meromorphic function and let

$$
\mathcal{R}^{\#}(g)=\frac{\sum_{i=1}^{n} a_{i} g^{i}}{\sum_{j=1}^{m} b_{j} g^{j}},
$$

be an irreducible rational function in $g$ with constant coefficients $\left\{a_{i}\right\},\left\{b_{j}\right\}$, where $a_{n} \neq 0$ and $b_{m} \neq 0$. Then

$$
T\left(r, \mathcal{R}^{\#}(g)\right)=\max \{n, m\} T(r, g)+S(r, g) .
$$

Lemma 3.2. [10] Let $\mathcal{P}(w)$ be a polynomial satisfying the property $(H)$. Then, $\mathcal{P}(w)$ is a uniqueness polynomial in a broad sense if and only if

$$
\sum_{1 \leq l<m \leq k} q_{l} q_{m}>\sum_{l=1}^{k} q_{l} .
$$

It can be easily verified that for the case $k \geq 4$, the condition (3.4) is always satisfied. Moreover, (3.4) holds when $\max \left\{q_{1}, q_{2}, q_{3}\right\} \geq 2$ for the case $k=3$ and when $\min \left\{q_{1}, q_{2}\right\} \geq 2$ and $q_{1}+q_{2} \geq 5$ for the case $k=2$.

\section{Proofs of the theorems}

In this section, we give the proofs of our main results.

Proof of Theorem 2.1. Let $f(z)$ and $f(z+c)$ be any two non-constant meromorphic functions. It is clear that

$$
\begin{aligned}
& \mathcal{F}^{\prime}=(f(z)-a)^{n}(f(z)-b)^{4} f^{\prime}(z) \text { and } \\
& \mathcal{G}^{\prime}=(f(z+c)-a)^{n}(f(z+c)-b)^{4} f^{\prime}(z+c) .
\end{aligned}
$$

We now discuss the following two cases:

Case 1. There exists a $\lambda>1, I \subset \mathbb{R}^{+}$with measure of $I$ as $+\infty$ such that

$$
\begin{aligned}
& 2 \bar{N}\left(r, \frac{1}{f(z)-a}\right)+2 \bar{N}\left(r, \frac{1}{f(z)-b}\right) \\
\geq & \lambda\{T(r, f(z))+T(r, f(z+c))\}+S(r, f(z))+S(r, f(z+c)),
\end{aligned}
$$

where $r \rightarrow+\infty, r \in I$. 
Let $\Phi$ is defined as in (3.3). Our aim is to show that $\Phi=0$. Let if possible $\Phi \not \equiv 0$. Then since $n \geq 4$, so from the construction of $\Phi$, we get

$$
4 \bar{N}\left(r, \frac{1}{f(z)-a}\right)+4 \bar{N}\left(r, \frac{1}{f(z)-b}\right) \leq N\left(r, \frac{1}{\Phi}\right) .
$$

The possible poles of $\Phi$ occur at the following points: (i) poles of $f(z)$, (ii) poles of $f(z+c)$, (iii) all the zeros of $\mathcal{F}$ of multiplicities $\geq 2$ and (iv) all the zeros of $\mathcal{G}$ of multiplicities $\geq 2$.

So we have

$$
N(r, \Phi) \leq \bar{N}(r . f(z))+\bar{N}_{(2}\left(r, \frac{1}{\mathcal{F}}\right)+\bar{N}(r . f(z+c))+\bar{N}_{(2}\left(r, \frac{1}{\mathcal{G}}\right) .
$$

By using First Fundamental Theorem and (4.2), (4.3), we get

$$
\begin{aligned}
& 4 \bar{N}\left(r, \frac{1}{f(z)-a}\right)+4 \bar{N}\left(r, \frac{1}{f(z)-b}\right) \\
\leq & N\left(r, \frac{1}{\Phi}\right) \\
\leq & N(r, \Phi) \\
\leq & \bar{N}(r . f(z))+\bar{N}_{(2}\left(r, \frac{1}{\mathcal{F}}\right)+\bar{N}(r . f(z+c))+\bar{N}_{(2}\left(r, \frac{1}{\mathcal{G}}\right) \\
& +S(r, f(z))+S(r, f(z+c)) .
\end{aligned}
$$

Again since $\bar{E}_{f(z)}\left(\mathcal{S}_{2}\right)=\bar{E}_{f(z+c)}\left(\mathcal{S}_{2}\right)$, so we must have

$$
\begin{aligned}
& \bar{N}\left(r, \frac{1}{f(z)-a}\right)+\bar{N}\left(r, \frac{1}{f(z)-b}\right) \\
= & \bar{N}\left(r, \frac{1}{f(z+c)-a}\right)+\bar{N}\left(r, \frac{1}{f(z+c)-b}\right) .
\end{aligned}
$$

Adding $\bar{N}\left(r, \frac{1}{\mathcal{F}}\right)+\bar{N}\left(r, \frac{1}{\mathcal{G}}\right)$ on both sides of (4.4), we get

$$
\begin{aligned}
& 4 \bar{N}\left(r, \frac{1}{f(z)-a}\right)+4 \bar{N}\left(r, \frac{1}{f(z)-b}\right)+\bar{N}\left(r, \frac{1}{\mathcal{F}}\right) \\
+ & \bar{N}\left(r, \frac{1}{\mathcal{G}}\right) \\
\leq & \bar{N}(r \cdot f(z))+N\left(r, \frac{1}{\mathcal{F}}\right)+\bar{N}(r \cdot f(z+c))+N\left(r, \frac{1}{\mathcal{G}}\right) \\
& +S(r, f(z))+S(r, f(z+c)) .
\end{aligned}
$$


Next using (4.5) in (4.6), we get

$$
\begin{aligned}
& 2\left\{\bar{N}\left(r, \frac{1}{f(z)-a}\right)+\bar{N}\left(r, \frac{1}{f(z)-b}\right)\right\} \\
& \left\{\bar{N}\left(r, \frac{1}{f(z)-a}\right)+\bar{N}\left(r, \frac{1}{f(z)-b}\right)\right\}+\bar{N}\left(r, \frac{1}{\mathcal{F}}\right)+\bar{N}\left(r, \frac{1}{\mathcal{G}}\right) \\
& +\left\{\bar{N}\left(r, \frac{1}{f(z+c)-a}\right)+\bar{N}\left(r, \frac{1}{f(z+c)-b}\right)\right\} \\
\leq \quad & \bar{N}(r \cdot f(z))+N\left(r, \frac{1}{\mathcal{F}}\right)+\bar{N}(r . f(z+c))+N\left(r, \frac{1}{\mathcal{G}}\right)+S(r, f(z)) \\
+ & S(r, f(z+c)) .
\end{aligned}
$$

By applying Second Fundamental Theorem, we get

$$
\begin{aligned}
& (n+5)\{T(r, f(z))+T(r, f(z+c))\} \\
\leq & \bar{N}\left(r, \frac{1}{\mathcal{F}}\right)+\bar{N}\left(r, \frac{1}{f(z)-a}\right)+\bar{N}\left(r, \frac{1}{f(z)-b}\right)+\bar{N}\left(r, \frac{1}{\mathcal{G}}\right) \\
& +\bar{N}\left(r, \frac{1}{f(z+c)-a}\right)+\bar{N}\left(r, \frac{1}{f(z+c)-b}\right)+S(r, f(z)) \\
+ & S(r, f(z+c)) .
\end{aligned}
$$

Adding

$$
2 \bar{N}\left(r, \frac{1}{f(z)-a}\right)+2 \bar{N}\left(r, \frac{1}{f(z)-b}\right)
$$

both sides in (4.8) and using (4.7), we get

$$
\begin{aligned}
& (n+5)\{T(r, f(z))+T(r, f(z+c))\}+2 \bar{N}\left(r, \frac{1}{f(z)-a}\right) \\
& +2 \bar{N}\left(r, \frac{1}{f(z)-b}\right) \\
\leq & N\left(r, \frac{1}{\mathcal{F}}\right)+N\left(r, \frac{1}{\mathcal{G}}\right)+\bar{N}(r, f(z))+\bar{N}(r, f(z+c)) \\
& +S(r, f(z))+S(r, f(z+c)) \\
\leq \quad & (n+6)\{T(r, f(z))+T(r, f(z+c))\} .
\end{aligned}
$$

i.e.,

$$
2 \bar{N}\left(r, \frac{1}{f(z)-a}\right)+2 \bar{N}\left(r, \frac{1}{f(z)-b}\right) \leq\{T(r, f(z))+T(r, f(z+c))\},
$$

which is not possible for $\lambda>1$ in view of (4.1).

Thus, we get $\Phi \equiv 0$. i.e., $\mathcal{F} \equiv \mathcal{A G}$, for $\mathcal{A} \in \mathbb{C} \backslash\{0\}$. Using Lemma 3.1, we have

$$
T(r, f(z))=T(r, f(z+c))+S(r, f(z)) .
$$


Subcase 1.1. Let $\mathcal{A} \neq 1$.

So from the relation $\mathcal{F} \equiv \mathcal{A G}$, we get

$$
\mathcal{F}-\mathcal{A} \equiv \mathcal{A}(\mathcal{G}-1) .
$$

A simple calculation shows that the polynomial $\mathcal{P}(z)-\mathcal{A}$ has all simple distinct roots and let them be $\sigma_{j}(j=1,2, \ldots, n+5)$ and all $\sigma_{j} \neq a, b$. Also we note that the polynomial $\mathcal{P}(z)-1$ has roots as $a$ of multiplicity $n+1$ and rest are $\delta_{j}(j=1,2,3,4)$. Thus we see from (4.10) that

$$
\begin{aligned}
& \sum_{j=1}^{n+5} \bar{N}\left(r, \frac{1}{f(z)-\sigma_{j}}\right) \\
= & \bar{N}\left(r, \frac{1}{f(z+c)-a}\right)+\sum_{j=1}^{4} \bar{N}\left(r, \frac{1}{f(z+c)-\delta_{j}}\right) .
\end{aligned}
$$

By applying Second Fundamental Theorem and (4.9), we have

$$
\begin{aligned}
& (n+3) T(r, f(z)) \\
\leq & \sum_{j=1}^{n+5} \bar{N}\left(r, \frac{1}{f(z)-\sigma_{j}}\right)+S(r, f(z)) \\
\leq & \bar{N}\left(r, \frac{1}{f(z+c)-a}\right)+\sum_{j=1}^{4} \bar{N}\left(r, \frac{1}{f(z+c)-\delta_{j}}\right)+S(r, f(z)) \\
\leq & 5 T(r, f(z))+S(r, f(z)),
\end{aligned}
$$

which contradicts $n \geq 4$.

Subcase 1.2. Let $\mathcal{A}=1$. i.e., we have $\mathcal{F} \equiv \mathcal{G}$. Thus we get $\mathcal{P}(f) \equiv \mathcal{P}(f(z+c))$. We see that the polynomial $\mathcal{P}(z)=\int_{0}^{z-a}(t-a)^{n}(t-b)^{4} d t+1$ satisfies the condition $(\mathrm{H})$ and (3.4) since $\mathcal{P}^{\prime}(z)=(z-a)^{n}(z-b)^{4}, k=2, e_{1}=a, e_{2}=b$ and $q_{1}=n \geq 4, q_{2}=4$. We next see that $\min \left\{q_{1}, q_{2}\right\}=\min \{n, 4\} \geq 2$ and $q_{1}+q_{2}=n+4 \geq 5$. Therefore by Lemma 3.2 , we see that the polynomial $\mathcal{P}(z)$ is a uniqueness polynomial in a broad sense. Hence the relation $\mathcal{P}(f) \equiv \mathcal{P}(f(z+c))$ implies $f(z) \equiv f(z+c)$.

Case 2. There exists $I \subset \mathbb{R}^{+}$such that measure of $I$ is $+\infty$ such that

$$
\begin{aligned}
& 2 \bar{N}\left(r, \frac{1}{f(z)-a}\right)+2 \bar{N}\left(r, \frac{1}{f(z)-b}\right) \\
\leq & \left(1+\frac{1}{1000}\right)\{T(r,(z) f)+T(r, f(z+c))\}+S(r,(z) f)+S(r, f(z+c) .
\end{aligned}
$$

We claim that $\mathcal{H} \equiv 0$. Suppose that $\mathcal{H} \not \equiv 0$. Next in view of the definition $\mathcal{H}$, we see that

$$
\bar{N}_{1)}^{E}\left(r, \frac{1}{\mathcal{F}}\right)=\bar{N}_{1)}^{E}\left(r, \frac{1}{\mathcal{G}}\right) \leq N\left(r, \frac{1}{H}\right) .
$$

We see that the possible poles of $\mathcal{H}$ occur at the following points: (i) poles of $f(z)$, (ii) poles of $f(z+c)$, (iii) zeros of $f(z)$, (iv) 1-points of $f(z)$, (v) all those zeros of 
$f^{\prime}(z)$ which are not the zeros of $f(z)(f(z)-1)$ and (vi) all those zeros of $f^{\prime}(z+c)$ which are not the zeros of $f(z+c)(f(z+c)-1)$. Thus we get

$$
\begin{aligned}
N(r, \mathcal{H}) & \leq \bar{N}(r, f(z))+\bar{N}\left(r, \frac{1}{f(z)-a}\right)+\bar{N}\left(r, \frac{1}{f(z)-b}\right) \\
& +\bar{N}(r, f(z+c))+\bar{N}_{0}\left(r, 0 ; f^{\prime}(z)\right)+\bar{N}_{0}\left(r, 0 ; f^{\prime}(z+c)\right),
\end{aligned}
$$

where $\bar{N}_{0}\left(r, \frac{1}{f^{\prime}(z)}\right)$ is the reduced counting function of all those zeros of $f^{\prime}(z)$ which are not the zeros of $(f(z)-a)(f(z)-b)$. Similarly $\bar{N}_{0}\left(r, \frac{1}{f^{\prime}(z+c)}\right)$ is defined.

Therefore using First Fundamental Theorem, we get

$$
\begin{aligned}
\bar{N}_{1)}^{E}\left(r, \frac{1}{\mathcal{F}}\right) r & \leq N\left(r, \frac{1}{\mathcal{H}}\right) \\
& \leq N(r, \mathcal{H}) \\
& \leq \bar{N}(r, f(z))+\bar{N}\left(r, \frac{1}{f(z)-a}\right)+\bar{N}\left(r, \frac{1}{f(z)-b}\right) \\
& +\bar{N}(r, f(z+c))+\bar{N}_{0}\left(r, 0 ; f^{\prime}(z)\right)+\bar{N}_{0}\left(r, 0 ; f^{\prime}(z+c)\right) .
\end{aligned}
$$

We also note that

$$
\bar{N}_{(2}\left(r, \frac{1}{\mathcal{F}}\right) \leq \bar{N}_{0}\left(r, \frac{1}{f^{\prime}(z)}\right), \bar{N}_{(2}\left(r, \frac{1}{\mathcal{G}}\right) \leq \bar{N}_{0}\left(r, \frac{1}{f^{\prime}(z+c)}\right) .
$$

We define

$$
\Psi(z):=\frac{f^{\prime}(z)}{[f(z)-a][(f(z)-b]} \frac{f^{\prime}(z+c)}{[f(z+c)-a][f(z+c)-b} .
$$

From the definition of $\Psi$ and by using First Fundamental Theorem and (4.5), we get

$$
\begin{aligned}
& N_{0}\left(r, \frac{1}{f^{\prime}(z)}\right)+N_{0}\left(r, \frac{1}{f^{\prime}(z+c)}\right) \\
\leq & \bar{N}\left(r, \frac{1}{\Psi}\right) \\
\leq & \bar{N}(r, \Psi) \\
\leq & \bar{N}\left(r, \frac{1}{f(z)-a}\right)+\bar{N}\left(r, \frac{1}{f(z)-b}\right)+\bar{N}\left(r, \frac{1}{f(z+c)-a}\right) \\
& +\bar{N}\left(r, \frac{1}{f(z+c)-b}\right)+S(r, f(z))+S(r, f(z+c)) \\
\leq & 2 \bar{N}\left(r, \frac{1}{f(z)-a}\right)+2 \bar{N}\left(r, \frac{1}{f(z)-b}\right)+S(r, f(z))+S(r, f(z+c)) .
\end{aligned}
$$

Adding

$$
\bar{N}_{(2}\left(r, \frac{1}{\mathcal{F}}\right)+\bar{N}_{(2}\left(r, \frac{1}{\mathcal{G}}\right)+\bar{N}\left(r, \frac{1}{f(z)-a}\right)+\bar{N}\left(r, \frac{1}{f(z)-b}\right)
$$


both sides of (4.15), we get

$$
\begin{aligned}
& \bar{N}_{1)}^{E}\left(r, \frac{1}{\mathcal{F}}\right)+\bar{N}_{(2}\left(r, \frac{1}{\mathcal{F}}\right)+\bar{N}_{(2}\left(r, \frac{1}{\mathcal{G}}\right)+\bar{N}\left(r, \frac{1}{f(z)-a}\right) \\
+ & \bar{N}\left(r, \frac{1}{f(z)-b}\right) \\
\leq & \bar{N}(r, f(z))+2 \bar{N}\left(r, \frac{1}{f(z)-a}\right)+2 \bar{N}\left(r, \frac{1}{f(z)-b}\right)+\bar{N}(r, f(z+c)) \\
+ & 2 \bar{N}_{0}\left(r, \frac{1}{f^{\prime}(z)}\right)+2 \bar{N}_{0}\left(r, \frac{1}{f^{\prime}(z+c)}\right) .
\end{aligned}
$$

i.e.,

$$
\begin{aligned}
& \bar{N}\left(r, \frac{1}{\mathcal{F}}\right)+\bar{N}\left(r, \frac{1}{f(z)-a}\right)+\bar{N}\left(r, \frac{1}{f(z)-b}\right) \\
\leq & \bar{N}(r, f(z))+6 \bar{N}\left(r, \frac{1}{f(z)-a}\right)+6 \bar{N}\left(r, \frac{1}{f(z)-b}\right)+\bar{N}(r, f(z+c)) \\
+ & S(r, f(z))+S(r, f(z+c)) .
\end{aligned}
$$

Similarly, we get

$$
\begin{aligned}
& \bar{N}\left(r, \frac{1}{\mathcal{G}}\right)+\bar{N}\left(r, \frac{1}{f(z+c)-a}\right)+\bar{N}\left(r, \frac{1}{f(z+c)-b}\right) \\
\leq & \bar{N}(r, f(z+c))+6 \bar{N}\left(r, \frac{1}{f(z+c)-a}\right)+6 \bar{N}\left(r, \frac{1}{f(z+c)-b}\right) \\
+ & \bar{N}(r, f(z))+S(r, f(z))+S(r, f(z+c)) .
\end{aligned}
$$

By applying Second Fundamental Theorem and (4.12), (4.18) and (4.19), we get

$$
\begin{aligned}
& (n+5)\{T(r, f(z))+T(r, f(z+c))\} \\
\leq & \bar{N}\left(r, \frac{1}{\mathcal{F}}\right)+\bar{N}(r, f(z))+\bar{N}\left(r, \frac{1}{f(z)-a}\right)+\bar{N}\left(r, \frac{1}{\mathcal{G}}\right)+\bar{N}(r, f(z+c)) \\
& +\bar{N}\left(r, \frac{1}{f(z+c)-a}\right)+S(r, f(z))+S(r, f(z+c)) \\
\leq & 2 \bar{N}(r, f(z))+2 \bar{N}(r, f(z+c))+6 \bar{N}\left(r, \frac{1}{f(z)-a}\right) \\
+ & 6 \bar{N}\left(r, \frac{1}{f(z+c)-a}\right)+6 \bar{N}\left(r, \frac{1}{f(z)-b}\right)+6 \bar{N}\left(r, \frac{1}{f(z+c)-b}\right) \\
+ & S(r, f(z))+S(r, f(z+c)) \\
\leq & \left(8+\frac{6}{1000}\right)\{T(r, f(z))+T(r, f(z+c))\}++S(r, f(z))+S(r, f(z+c)),
\end{aligned}
$$

which contradicts $n \geq 4$. 
Therefore, we have $\mathcal{H} \equiv 0$. Thus we get

$$
\frac{1}{\mathcal{F}} \equiv \frac{\mathcal{A}}{\mathcal{G}}+\mathcal{B}
$$

where $\mathcal{A}(\neq 0), \mathcal{B} \in \mathbb{C}$. In view of Lemma 3.1, we see from (4.20) that

$$
T(r, f(z))=T(r, f(z+c))+S(r, f(z)) .
$$

Subcase 2.1. Let $\mathcal{B} \neq 0$. Thus we must have

$$
\bar{N}(r, f(z))=\bar{N}(r, \mathcal{F})=\bar{N}\left(r, \frac{1}{\mathcal{G}+\frac{\mathcal{A}}{\mathcal{B}}}\right) \geq 3 T(r, f(z+c))+S(r, f(z+c)),
$$

which is absurd in view of (4.21).

Subcase 2.2. So we have $\mathcal{B}=0$. Therefore (4.20) reduces to $\mathcal{G}=\mathcal{A} \mathcal{F}$. Proceeding exactly same way as done in Subcase 1.1, we get $f(z) \equiv f(z+c)$.

Proof of Theorem 2.2. Since $f(z)$ is a non-constant entire function, so we must have $\bar{N}(r, f(z))=0$ and hence $\bar{N}(r, f(z+c))=0$. Now keeping this in mind, the rest of the proof follows the proof of Theorem 2.1.

\section{An open question}

Question 5.1. Is it possible to reduce the cardinalities further of two sets sharing problem (in case of IM sharing) for the periodicity of a meromorphic function $f$ ?

\section{References}

[1] Ahamed, M.B., An investigation on the conjecture of Chen and Yi, Results Math., 74(2019), 122.

[2] Banerjee, A., Uniqueness of meromorphic functions sharing two sets with finite weight, Port. Math., 65(2008), no. 1, 81-93.

[3] Banerjee, A., Uniqueness of meromorphic functions sharing two sets with finite weight II, Tamkang J. Math., 41(2010), no. 4, 379-392.

[4] Banerjee, A., Ahamed, M.B., Uniqueness of meromorphic function with its shift operator under the purview of two or three shared sets, Math. Slovaca, 69(2019), no. 3, 557-572.

[5] Bhoosnurmath, S.S., Dyavanal, R.S., Uniqueness of meromorphic functions sharing a set, Bull. Math. Anal. Appl., 3(2011), no. 3, 200-208.

[6] Bhoosnurmath, S.S., Kabbur, S.R., Value distribution and uniqueness theorems for difference of entire and meromorphic functions, Int. J. Anal. Appl., 2(2013), no. 2, 124-136.

[7] Chen, B., Chen, Z., Meromorphic functions sharing two sets with its difference operator, Bull. Malays. Math. Sci. Soc., 35(2012), no. 3, 765-774.

[8] Chen, B., Chen, Z., Li, S., Uniqueness of difference operators of meromorphic functions, J. Inequal. Appl., 48(2012), 1-19.

[9] Fang, M., Lahiri, I., Unique range set for certain meromorphic functions, Indian J. Math., 45(2003), no. 2, 141-150. 
[10] Fujimoto, H., On uniqueness polynomials for meromorphic functions, Nagoya Math. J., 170(2003), 33-46.

[11] Goldberg, A., Ostrovskii, I., Value Distribution of Meromorphic Functions, Transl. Math. Monogr., vol. 236, American Mathematical Society, Providence, RI, 2008, translated from the 1970 Russian original by Mikhail Ostrovskii, with an appendix by Alexandre Eremenko and James K. Langley.

[12] Gross, F., Factorization of meromorphic functions and some open problems, Proc. Conf. Univ. Kentucky, Lexington, Ky(1976); Complex Analysis, Lecture Notes in Math., 599(1977), 51-69, Springer Verlag.

[13] Hayman, W.K., Meromorphic Functions, The Clarendon Press, Oxford, 1964.

[14] Laine, I., Nevanlinna Theory and Complex Differential Equations, Walter de Gruyter, Berlin, 1993.

[15] Mokhonako, A.Z., On the Nevanlinna characteristics of some meromorphic functions, Theo. Funct. Funct. Anal. Appl., Izd-vo Kharakovsk Un-ta, 14(1971), 83-87.

[16] Nevanlinna, R., Le Théorème de Picard-Borel et la Théorie des Fonctions Méromorphes, Gauthiers-Villars, Paris, 1929.

[17] Qi, X.G., Dou, J., Yang, L.Z., Uniqueness and value distribution for difference operator of meromorphic function, Adv. Difference Equ., 32(2012), 1-9.

[18] Yang, C.C., Yi, H.X., Uniqueness Theory of Meromorphic Functions, Math. Appl., 557(2003), Kluwer Academic Publishers Group, Dordrecht.

[19] Yi, H.X. Lin, W.C., Uniqueness of meromorphic functions and a question of Gross, Kyungpook Math. J., 46(2006), 437-444.

[20] Zhang, J.L., Value distribution and sets of difference of meromorphic functions, J. Math. Anal. Appl., 367(2010), no. 2, 401-408.

[21] Zhang, J., Xu, Y., Meromorphic functions sharing two sets, Appl. Math. Lett., 21(2008), 471-476.

Molla Basir Ahamed

Department of Mathematics

Kalipada Ghosh Tarai Mahavidyalaya

Bagdogra, Darjeeling

West Bengal, 734014, India

e-mail: basir_math_kgtm@yahoo.com, bsrhmd2014@gmail.com. 